\title{
Modelling the heat dynamics of a monitored Test Reference Environment for Building Integrated Photovoltaic systems using stochastic differential equations
}

\author{
C. Lodi ${ }^{\text {a,* }}$, P. Bacher ${ }^{\text {b }}$ J. Cipriano ${ }^{\mathrm{c}}$, H. Madsen $^{\mathrm{b}}$ \\ a Applied Physics Section of the Environment Science Department, University of Lleida, c/Jaume II 69, 25001 Lleida, Spain \\ b IMM, Technical University of Denmark, Richard Pedersen Plads, Building 305, 2800 Lyngby, Denmark \\ ${ }^{\mathrm{c}}$ CIMNE, Building Energy and Environment Group, c/Dr Ulles 2, 08224 Terrassa, Spain
}

\section{A R T I C L E I N F O}

\section{Article history:}

Received 14 January 2012

Received in revised form 8 March 2012

Accepted 25 March 2012

\section{Keywords:}

BIPV systems

Forced convection

Grey-box modelling

Parameter identification

\begin{abstract}
A B S T R A C T
This paper deals with grey-box modelling of the energy transfer of a double skin Building Integrated Photovoltaic (BIPV) system. Grey-box models are based on a combination of prior physical knowledge and statistics, which enable identification of the unknown parameters in the system and accurate prediction of the most influential variables. The experimental data originates from tests carried out with an airbased BIPV system installed in a Test Reference Environment. BIPV systems represent an interesting application for achieving the requirements of the EU EPBD Directive. Indeed, these systems could reduce the ventilation thermal losses of the building by pre-heating the fresh air. Furthermore, by decreasing PV module temperature, the ventilation air heat extraction can simultaneously increase electrical and thermal energy production of the building. A correct prediction of the PV module temperature and heat transfer coefficients is fundamental in order to improve the thermo-electrical production.

The considered grey-box models are composed of a set of continuous time stochastic differential equations, holding the physical description of the system, combined with a set of discrete time measurement equations, which represent the data driven part.

In the present work, both one-state and two-state non-linear grey-box models are considered. In order to validate the results, the residuals are analysed for white-noise properties.
\end{abstract}

(C) 2012 Elsevier B.V. All rights reserved.

\section{Introduction}

The aim of the present work is to carry out an energy transfer characterization of an air-based double skin Building Integrated Photovoltaic (BIPV) system. One of the encompassing results of this work is to model the effect of the PV module temperature in order to optimize BIPV installations under forced convection where the ventilation air is used for pre-heating the incoming air. The implementation of the EU EPBD Directive and the prEN 13779 has fixed considerable air renovations per hour for non-residential buildings, which results in an inevitable increase in the energy consumption, especially during the winter season. One possibility to reduce these ventilation thermal losses is the installation of BIPV systems in which the fresh air is pre-heated. These systems can thus substitute or be combined with the heat recovery unit of the building to take advantage of the waste heat. Since mechanical ventilation systems are normally installed in non-residential buildings, forced ventilation regimes are analysed in this work.

\footnotetext{
* corresponding author. Tel.: +34 973003574 .

E-mail addresses: chiara.lodi@macs.udl.cat (C. Lodi), pb@imm.dtu.dk(P. Bacher), cipriano@cimne.upc.edu (J. Cipriano),hm@imm.dtu.dk (H. Madsen).
}

Reliable and detailed experimental data of air-based BIPV systems should be available for modelling purposes. During the PVHybrid-PAS EU project [1] a standard scheme for the performance evaluation of hybrid PV building components was developed. Within the IMPACT EU project [2] a common outdoor Test Reference Environment (TRE) [3] has been developed by the EU Joint Research Centre (JRC) in Ispra in order to assess the thermal exchange of the PV module with its environment. TRE is a standard outdoor facility for testing building integrated PV ventilated modules under forced regimes. With the experience gained from TRE experimental work, an improved version (Test Reference Environment of Lleida (TRE-L) as shown in Fig. 1) [4] was designed and built in the Lleida Outdoor Test centre (LOTCE). Several experiments, at different inclinations and ventilation regimes, have been performed and the initial experimental results are reported in [5]. Since TRE-L test facility is well insulated behind and on the lateral sides of the air channel, the effect of different rear-facing materials over the system can also be evaluated.

Different authors [6-8] have modelled the energy transfer of BIPV systems with continuous-discrete stochastic state space models based on experimental data. Grey-box modelling, based on stochastic differential equations (SDE's), is a well proven and promising method for describing the heat dynamics of buildings 


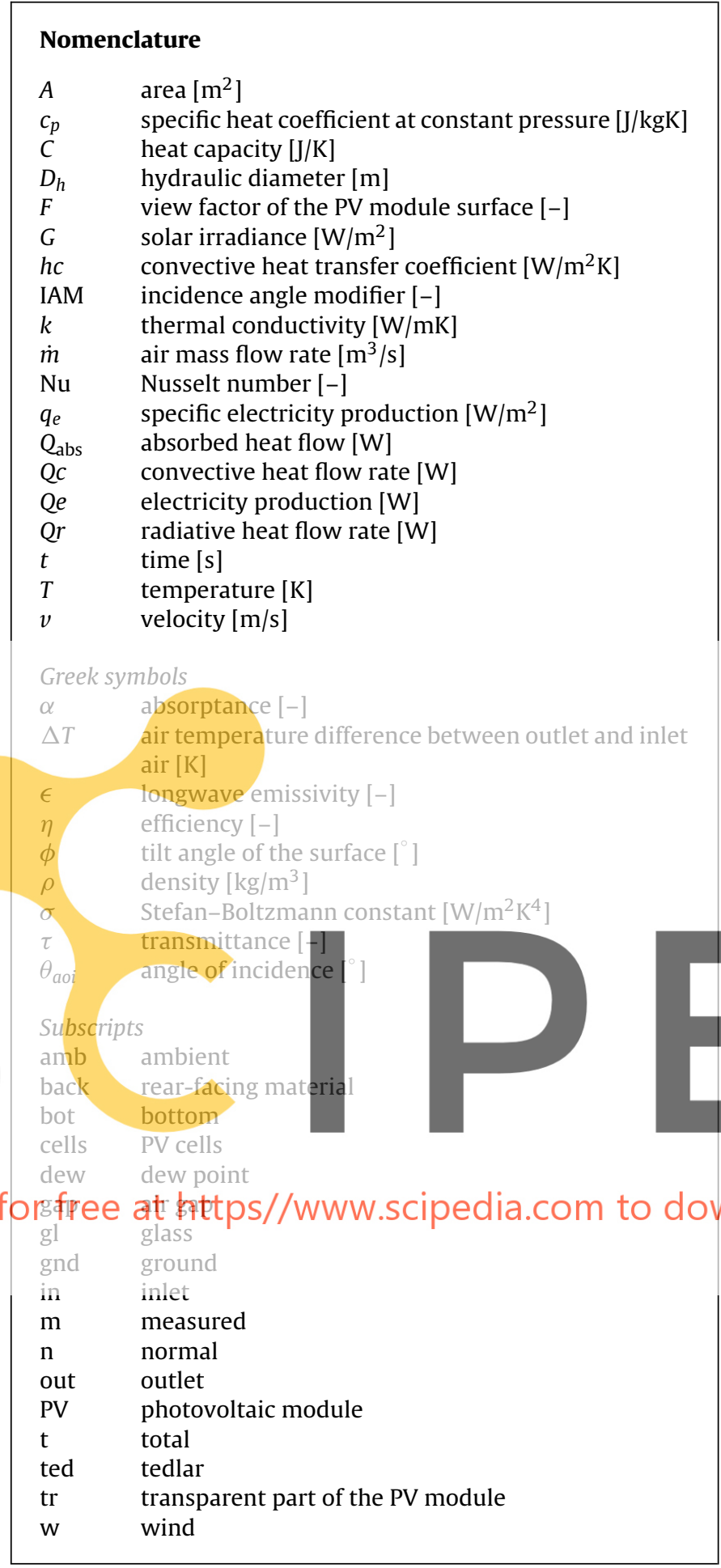

and components [9]. These dynamic models allow parameter estimation (system identification) and accurate prediction of the most influential variables. Grey-box models are composed by a set of stochastic differential equations, combined with a set of discrete time measurement equations. The SDE's hold the physical description of the system while the measurement equations represent the data driven part. One of the advantages of these models is the possibility to decompose the system noise into process noise and measurement noise. This allows for estimation of the unknown parameters in a prediction error (PE) setting as opposed to the common output error (OE) setting [6]. The models can be used for simulation, prediction and control applications.

Previous grey-box modelling work with TRE data from JRC was carried out by Jiménez et al. [6]. Within this work a non-linear model was found to be most suitable to describe the system and the authors emphasized that it was not possible to directly estimate the unknown physical parameters without more detailed measurements. In [7] the model formulated in [6] was applied with different experimental data from TRE prototype where transversal fins were placed in the air gap. The model described the experimental data satisfactory, but the authors suggested that an extension of the model from single-state to multiple-state might improve the performance of the model.

The grey-box models proposed in the present paper can be considered as an extension to the models described in [6,7]; the most relevant differences with the previous work is the direct estimation of the convective heat transfer coefficients between the PV module and the air gap, and the PV module heat capacity described through a single and a two-state model formulations. In addition, other physical inputs and outputs are also considered in the presented models: the angular dependency of the optical properties of the PV module, the electricity production, the effect of PV module inclination over the heat transfer coefficients and the consideration of the ground and sky temperatures for the radiative losses calculations. The estimated convective heat transfer coefficients within the air gap are also compared with the coefficients calculated from [10-14].

The paper starts with a description of the experimental set-up and data. Then follows a section about the modelling approach where the grey-box models structure and the considered models are presented. The following section summarizes the results of the modelling work: starting with a description of the estimated parameters, followed by the model evaluation in tine and frequency domain and followed by a comparison of the estimated Nusselt numpers with literature relations. Finally conclusions are drawn and further developments

(1) Experimental set-up thed.

\subsection{Rescription of the set-up .}

the version without the watermark

The TRE-L prototype (see Fig. 1) is composed by a thermally well insulated wooden box (with external sizes of $2.06 \times 2.36 \times 0.37 \mathrm{~m}$ ) and a support structure which allows any inclination to be tested. The wooden box is filled with a $0.2 \mathrm{~m}$ thick expanded polystyrene (EPS) layer and the walls are formed by $0.02 \mathrm{~m}$ thick plywood painted with white varnish to minimize solar absorption. The prototype has a south facing opening where a glass-tedlar monocrystalline-Si PV module (with dimensions of $0.976 \times 1.507 \mathrm{~m}$ ) is positioned. The air channel behind the PV module is $0.115 \mathrm{~m}$ wide and its cross sectional area is $0.112 \mathrm{~m}^{2}$. The hydraulic diameter is $0.198 \mathrm{~m}$. The air enters from the bottom and is extracted from the top by means of a $0.125 \mathrm{~m}$ diameter PVC tube, placed at the rear side of the box so that it remains shaded. A variable speed fan controls the airflow at several rates. An array of plastic made cylindrical tubes with a diameter of $0.005 \mathrm{~m}$ is placed at the inlet to guarantee a non disturbed pattern flow. The PV module can easily be removed in order to access the rear side of the air gap. This allows different rear-facing materials to be tested. In the experiments used for this analysis an ALANOD-Mirotherm absorber sheet was positioned. This Mirotherm absorber is black coloured and it has a solar absorption of $90 \pm 1 \%$ (in the range of wavelength between 380 and $1650 \mathrm{~nm}$ ) and a thermal emission of $5 \pm 2 \%$ (in the range of wavelength between 3 and $20 \mu \mathrm{m}$ ). The PV module is formed by 28 monocrystalline-Si solar cells ( $46 \%$ area covered by 
a

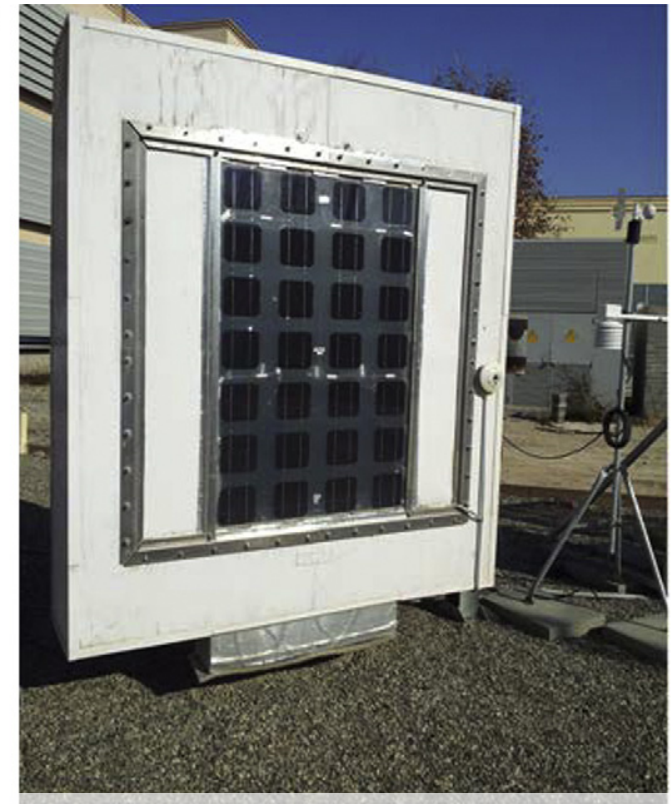

b

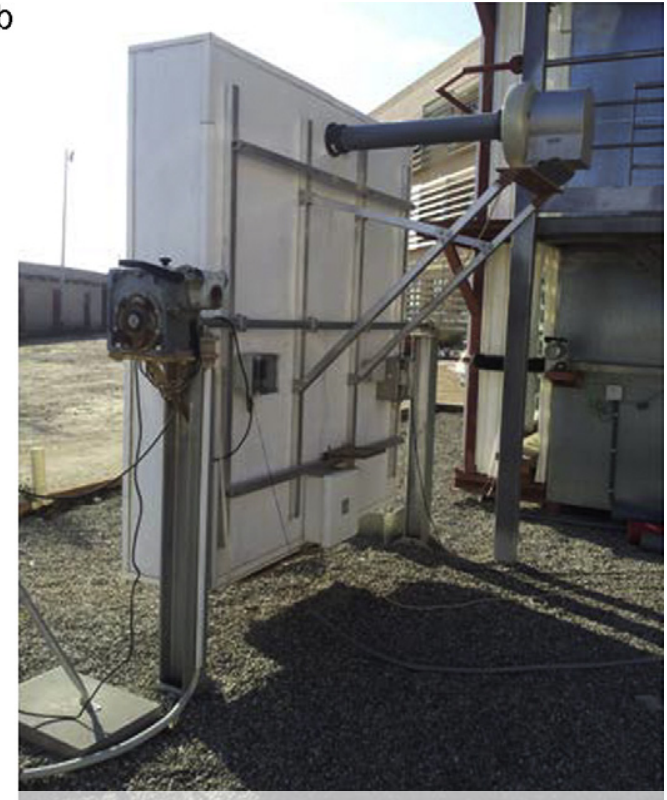

Fig. 1. Test Reference Environment of Lleida (TRE-L). (a) Front view; (b) rear view.

PV cells). In Fig. 2 an outline of the TRE-L measurement set-up is shown.

\subsection{Experimental data}

The data was collected in $30 \mathrm{~s}$ intervals and then averaged to 10 min values. The overall measurement period covers July to December 2010 and the trials consisted of 2-4 dayperiod tests. The following temperatures were measured: inlet and outlet temperatures within the air gap, surface temperature of the interior side of the PV module, and surface temperature of the black absorber sheet. The electrical energy production of each string of the PV module was recorded in the data logger. Wind direction and speed were recorded by a cup-type anemomete placed next to the TRE-I and at the same height; ambient temperature and humidity were colster forgfreat. at antet
sensors, see [5].

The monitoring campaign was extended over a half year period.

Tests on the TRE-L prototype were carried out with a fixed air gap width of $11.5 \mathrm{~cm}$, two different inclinations (vertical and $30^{\circ}$ ) and seven ventilation regimes. Experimental results from the first part of the monitoring campaign are summarized in [5]. Fig. 3 shows plots of measured PV module average temperature, air inlet and outlet temperatures, and solar radiation for different air flow rates in the air cavity. As demonstrated, both PV module and outlet air temperatures are strongly affected by variations in solar radiation. The dynamical response of both variables is delayed in time because of the system thermal inertia. This indicates that a description of the dynamics when modelling on this time resolytion is essential.

3. Energy transfer

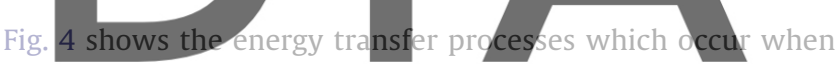
the PV ventilated module is exposed to solar radiation. The model takes on adiabatic conditions behind and to the lateral sides of the aidctheeversion without the watermark

The following heat transfer processes are considered within the system:

- Radiative heat losses to the exterior:
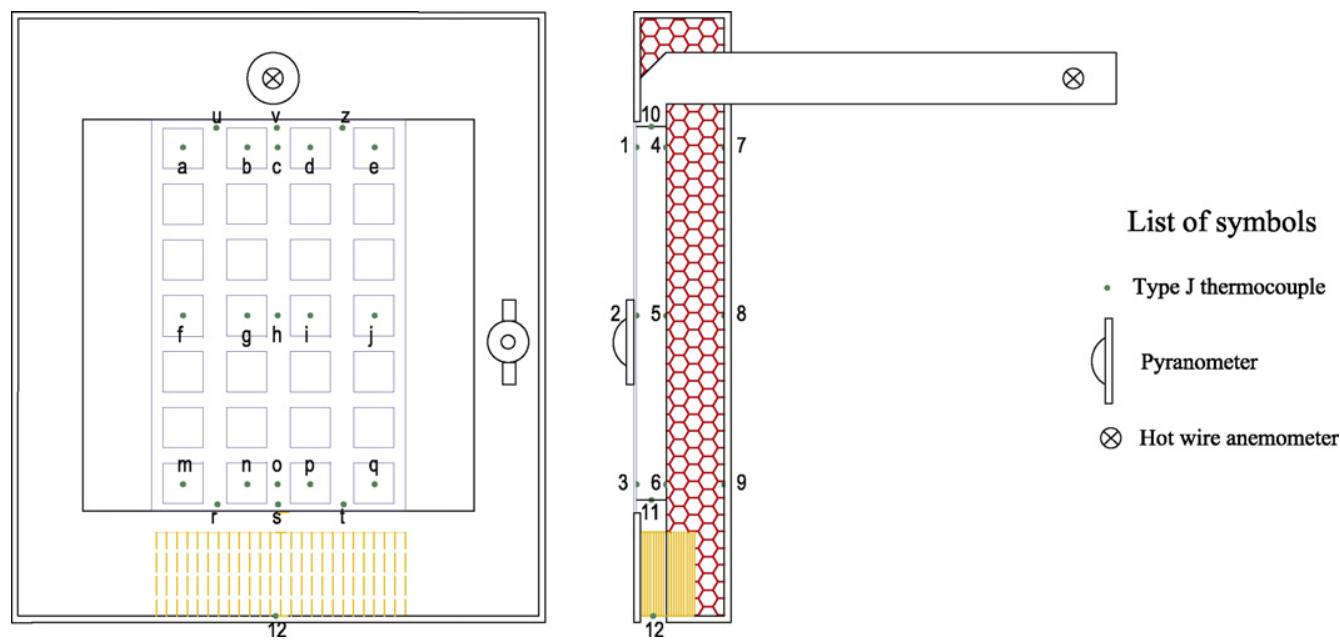

Fig. 2. Measurement set-up of the TRE-L prototype. 


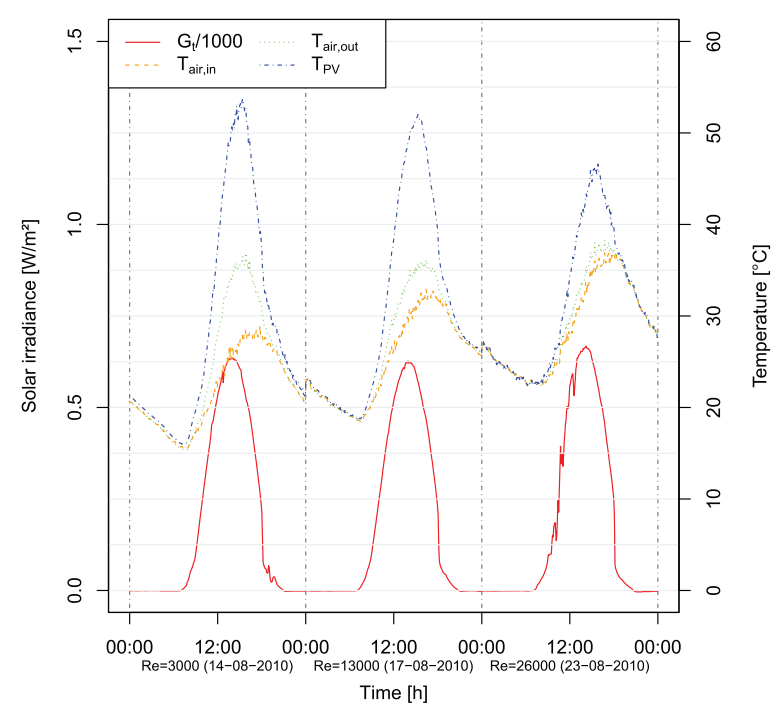

Fig. 3. Measured PV module average temperature, air inlet and outlet temperatures, and solar radiation for different air flow rates.

\section{$\mathrm{Qr}_{\mathrm{PV}, \mathrm{sky}}=A_{\mathrm{PV}} \sigma \epsilon_{\mathrm{gl}} F_{\mathrm{sky}}\left(T_{\mathrm{PV}}^{4}-T_{\mathrm{sky}}^{4}\right)+A_{\mathrm{PV}} \sigma \epsilon_{\mathrm{gl}} F_{\mathrm{gnd}}\left(T_{\mathrm{PV}}^{4}-T_{\mathrm{gnd}}^{4}\right)$}

- Convective heat transfer between the PV module and the exterior:

$Q c_{\mathrm{amb}}=h c_{\mathrm{PV}, \mathrm{amb}} A_{\mathrm{PV}}\left(T_{\mathrm{PV}}-T_{\mathrm{amb}}\right)$

- Solar radiation absorbed

duction:

$Q_{\text {abs }}=A_{\text {cells }}(\tau \alpha)_{n, \text { cells }} G_{t}$ IAM

$Q_{e}=A_{P V} q_{e}$

- Thermal radiative heat transfer between the PV module and the rear-facing material:
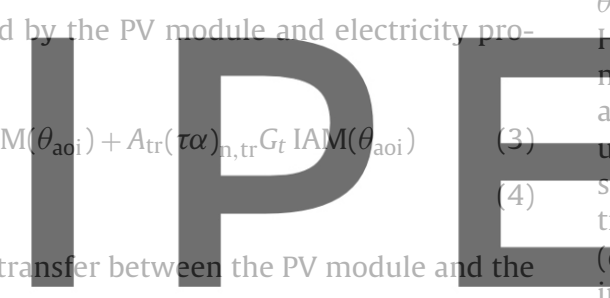

$$
\begin{aligned}
& Q c_{\text {gap }}=h c_{\mathrm{PV}, \text { gap }} A_{\mathrm{PV}}\left(T_{\mathrm{PV}}-T_{\text {gap }}\right)+h c_{\text {back,gap }} A_{\mathrm{PV}}\left(T_{\text {back }}-T_{\text {gap }}\right) \\
& \quad=\dot{m} \rho_{\text {gap }} c_{p} \Delta T
\end{aligned}
$$

These heat transfer processes are considered within the proposed models in Section 4.4 .

\section{Modelling approach}

\subsection{Model structure}

The grey-box models are continuous time stochastic state space models, which are lumped capacitance models with addition of noise. The evolution in time of the lumped states is described by a set of continuous time stochastic differential equations (SDE's) (system equations):

$d x_{t}=f\left(x_{t}, u_{t}, t, \theta\right) d t+\sigma\left(u_{t}, t, \theta\right) d \omega_{t}$

which are indirectly observed as described by the set of discrete time measurement equations (measurement equations):

$y_{k}=h\left(x_{k}, u_{k}, t_{k}, \theta\right)+e_{k}$

where $x_{t} \in \chi \subset \mathbb{R}^{n}$ is a vector of state variables, $u_{t} \in \mathcal{U} \subset \mathbb{R}^{m}$ is a vector of input variables, $t \in \mathbb{R}$ is the time variable, $\theta \in \Theta \subset \mathbb{R}^{p}$ is a vector of parameters, $y_{k} \in \mathcal{Y} \subset \mathbb{R}^{l}$ is a vector of output variables. $f(\cdot) \in \mathbb{R}^{n}, \sigma(\cdot) \in \mathbb{R}^{n \times n}$ and $h(\cdot) \in \mathbb{R}^{l}$ are known but possibly nonlinear functions; $\{\omega\}$ is an $n$-dimensional standard Wiener process. $\left\{e_{k}\right\}$ is an $l$-dimensional white noise process with $e_{k} \in N\left(0, S\left(u_{k}, t_{k}\right.\right.$, $\theta)$ ), and $\sigma($.$) is the gain of the increments of the Wiener process.$

Hence the total noise in the model is decomposed into a process se tern $\left(\varphi_{t}\right)$ and a measurement noise term $\left(e_{k}\right)$ and they are assumed to be mutually uncorrelated. This allows for estmation of nknown parameters from experimental data in aprediction error tting as op posed to the more commonly used butput en or setting [6]. The process noise accounts for: modelling approximations description of the dynamics, etc.), unrecognized and unnodeled inputs (notconsidered variables which may affect the system, etc.) and noise in the input measurements. The measurement noise term

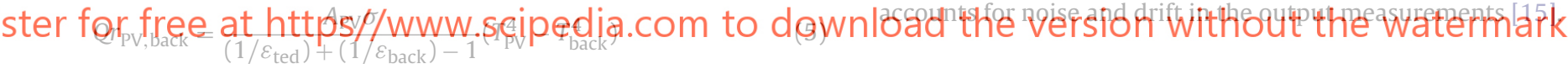

- Convective heat transfer within the air channel:

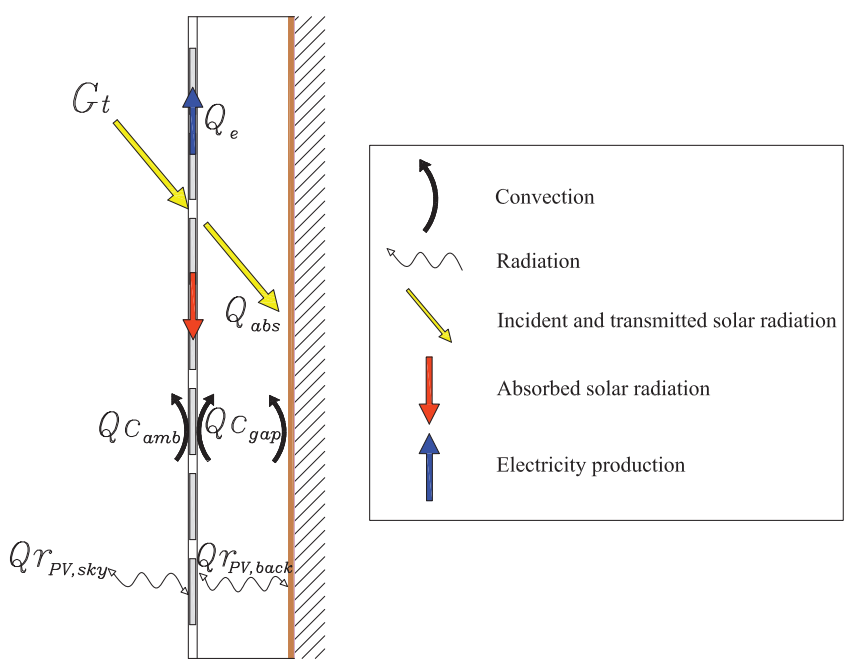

Fig. 4. Heat transfer processes within the TRE-L prototype.

\subsection{Parameter estimation}

The solution to Eq. (7) is a Markov process and unknown parameters of the model in Eq. (7) and (8) can be estimated with e.g., maximum likelihood or maximum a posteriori estimation [16]. Since no prior information about the parameters is available, maximum likelihood (ML) estimation is applied in the present modelling work. ML estimation of the unknown parameters is carried out by finding the parameters $\theta$ that maximize the likelihood function given a sequence of measurements $Y_{0}, Y_{1}, \ldots, Y_{N-1}, Y_{N}$. By introducing the notation:

$y_{N}=\left[Y_{0}, Y_{1}, \ldots, Y_{N-1}, Y_{N}\right]$

the likelihood function is the joint probability density:

$L\left(\theta ; y_{N}\right)=\left(\prod_{k=1}^{N} p\left(Y_{k} \mid \mathfrak{y}_{k-1}, \theta\right)\right) p\left(Y_{0} \mid \theta\right)$

where $p\left(Y_{k} \mid \mathfrak{y}_{k-1}, \theta\right)$ is a conditional density denoting the probability of observing $Y_{k}$ given the previous observations and the parameters $\theta . p\left(Y_{0} \mid \theta\right)$ is the probability distribution function (pdf) of the starting conditions. 

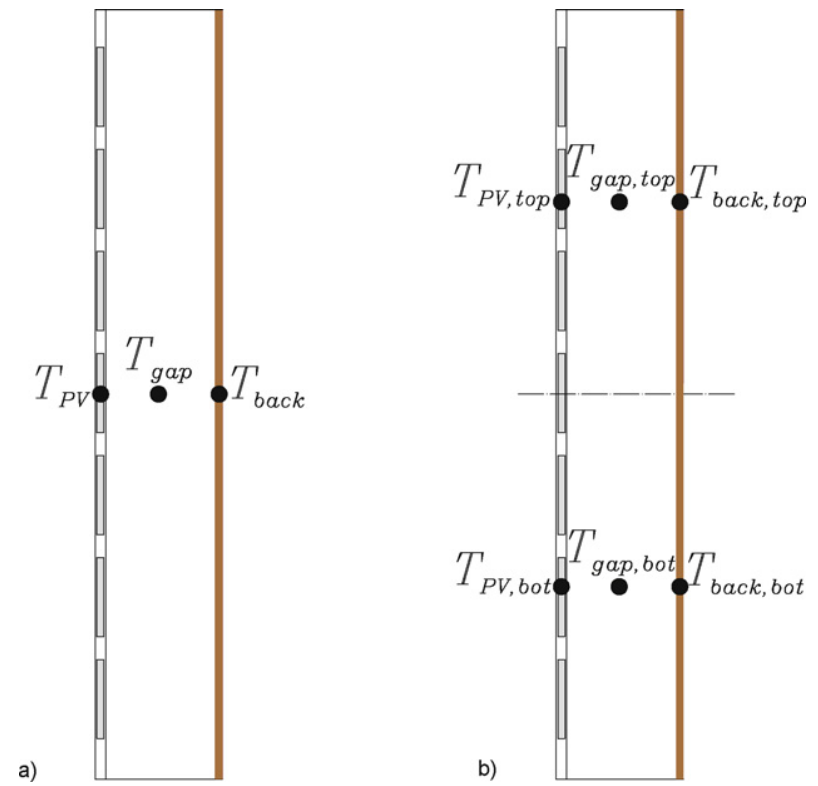

Fig. 5. Scheme of the estimation set-up. (a) Single-state model; (b) two-state model

The maximum likelihood estimates of the parameters are then given by:

$\hat{\theta}=\operatorname{argmax}\left\{L\left(\theta, y_{N}\right)\right\}$

The covariance matrix is obtained by approximating the Fisher Information matrix with the inverse of the observed Hessian matrix evaluated at the final estimates. The uncertainties of the parameter estimates are obtained by deconposing the covariance matrix into a diagonal matrix of the standard deviations of the parameter estimates and the corresponding co

4.3. Software implementation

The parameter estimation described in Section 4.2 has
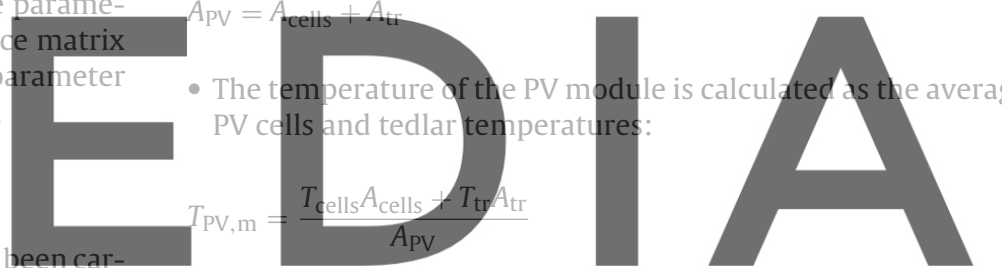
cied out using the software Continuous Time Stochastic Modelling CTSM [18]. CTSM is based on continuous-discrete stochastic state r fre की Kalman filter (EKF) algorithm [16] is applied to find maximum likelihood estimates of the parameters in the model.

\subsection{Considered grey-box models}

The presented grey-box models can be used for both simulation and forecasting of the PV module temperature, and for the direct estimation of the unknown physical parameters of the system. The PV module temperature is defined as both state and output variable of the models and it is assumed spatially uniform at any instant within each control volume (assumption of the lumped capacitance method [11]).

Prior research [6,7] has demonstrated that non-linear models are the most appropriate for describing the dynamics of the present system. Within the present work, non-linear single and two-state models are presented. In Fig. 5 a scheme of the estimation set-up for both models is shown.

\subsubsection{Single-state model formulation}

The considered single-state model predicts the average PV module temperature while estimating unknown parameters of the system. Since measurements of the output variable are required, the PV module temperature is obtained as the averaged temperatures of the transparent and opaque areas. The presented model
PV c
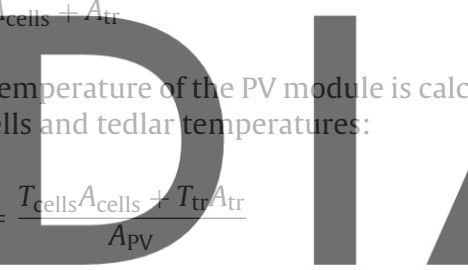

$(14)$

- The air gap temperature is calculated as a linear function of the

allows for an estimation of unknown physical parameters of the system (i.e. $h c_{\mathrm{PV} \text {,gap }}$ and $C_{\mathrm{PV}}$ ) and for statistically evaluating the accuracy of the estimates.

The single-state model for describing the heat dynamics of the PV module temperature in the TRE-L prototype can be expressed by the overall energy balance on the PV module surface:

$$
\begin{aligned}
& C_{\mathrm{PV}} d T_{\mathrm{PV}}=\left(A_{\mathrm{PV}} h c_{\mathrm{PV}, \mathrm{amb}}\left(T_{\mathrm{amb}}-T_{\mathrm{PV}}\right)+A_{\mathrm{PV}} h c_{\mathrm{PV}, \mathrm{gap}}\left(T_{\mathrm{gap}}-T_{\mathrm{PV}}\right)\right. \\
& +\frac{A_{\mathrm{PV}} \sigma}{\left(1 / \varepsilon_{\mathrm{ted}}\right)+\left(1 / \varepsilon_{\mathrm{back}}\right)-1}\left(T_{\mathrm{back}}^{4}-T_{\mathrm{PV}}^{4}\right)+A_{\mathrm{PV}} \sigma \epsilon_{\mathrm{gl}} F_{\mathrm{sky}}\left(T_{\mathrm{sky}}^{4}-T_{\mathrm{PV}}^{4}\right) \\
& +A_{\mathrm{PV}} \sigma \epsilon_{\mathrm{gl}} F_{\mathrm{gnd}}\left(T_{\mathrm{amb}}^{4}-T_{\mathrm{PV}}^{4}\right)+A_{\text {cells }}(\tau \alpha)_{\mathrm{n}, \mathrm{cells}} G_{t} \operatorname{IAM}\left(\theta_{\mathrm{aoi}}\right) \\
& \left.+A_{\mathrm{tr}}(\tau \alpha)_{\mathrm{n}, \mathrm{tr}} G_{t} \mathrm{IAM}\left(\theta_{\mathrm{aoi}}\right)-A_{\mathrm{PV}} q_{e}\right) d t+\sigma_{1} d \omega_{1}
\end{aligned}
$$

$T_{\mathrm{PV}, \mathrm{m}}=T_{\mathrm{PV}}+e$

where Eqs. (12) and (13) are the system and observation equations, Tectively.

The model has 10 inputs $\left(v_{w}, T_{\mathrm{amb}}, T_{\text {gap }}, T_{\text {back }}, F_{\text {sky }}, T_{\text {sky }}, F_{\text {gnd }}\right.$, $\left.\theta_{\text {aoi }}, G_{t}, q_{e}\right)$, estimated.

Several necessary inputs and parameters are calculated as follows:

- The PV module total area is the sum of the PV cells and the transparent areas:

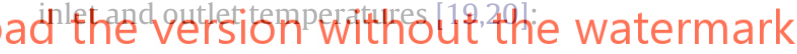

$T_{\text {gap }}=0.25 T_{\text {air, in }}+0.75 T_{\text {air, out }}$

Eq. (16) has been validated with air gap temperature measurements at mid-height of the TRE-L air gap.

- Since data of wind direction and speed is available, Sharples [21] relations are used for the exterior heat transfer coefficient, averaged for respectively windward and leeward directions:

$h c_{\mathrm{PV}, \mathrm{amb}}=3.72+1.16 v_{w}$

$h c_{\mathrm{PV}, \mathrm{amb}}=1.8+1.93 v_{\mathrm{w}}$

- For non-normal solar incidence, the incidence angle modifier $\operatorname{IAM}\left(\theta_{\text {aoi }}\right)$ is obtained by the expressions of Barker and Norton [22] for PV modules with clear glass:

$$
\begin{aligned}
& \operatorname{IAM}\left(\theta_{\text {aoi }}\right)=1-\left(3.310^{-3} \theta_{\text {aoi }}+4.1210^{-4} \theta_{\text {aoi }}^{2}+1.610^{-5} \theta_{\text {aoi }}^{3}\right. \\
& \left.\quad+2.610^{-7} \theta_{\text {aoi }}^{4}\right)
\end{aligned}
$$

- The longwave view factors of the PV module surface to respectively sky and ground surface are calculated as follows [23]:
8 known parameters (Apv, $A_{\text {cells }}, A_{\mathrm{tr}}, \epsilon_{\mathrm{ted}}, \epsilon_{\mathrm{back}}, \epsilon_{\mathrm{gl}},(\tau \alpha)_{\text {nn cells }}$, 
$F_{\text {sky }}=0.5(1+\cos \phi)$

$F_{\text {gnd }}=0.5(1-\cos \phi)$

- Since data of the dew point ambient temperature is available, the Duffie and Beckman relation [24] has been used for the sky temperature calculation:

$T_{\text {sky }}=T_{\mathrm{amb}}\left(0.711+0.0056 T_{\mathrm{dew}}+0.000073 T_{\mathrm{dew}}^{2}\right.$

$\left.+0.013 \cos \left(\pi t_{m} / 12\right)\right)$

where $t_{m}$ is the time from midnight in hours and the ground temperature $T_{\text {gnd }}$ is assumed to be the same as the ambient temperature. In previous works ([6,7]) the effective sky temperature was considered a steady-state parameter to estimate.

\subsubsection{Two-state model formulation}

Since the PV module, black absorber and air gap temperatures are collected at three different heights (see Fig. 2) and considerable differences (up to $10^{\circ} \mathrm{C}$ ) are found between top and bottom PV module temperatures, the single-state model has been extended to a two states formulation in order to get a better prediction. The need of using a multiple state model for the system description was expressed also in [7]. With the two-state model, top and bottom PV module average temperatures are estimated separately, taking the temperature gradient over the vertical axis into account. The two-state model is formulated as follows:

$\frac{C_{\mathrm{PV}}}{2} d T_{\text {PVbot }}=\left(\frac{A_{\mathrm{PV}}}{2} h c_{\mathrm{PV}, \mathrm{amb}}\left(T_{\mathrm{amb}}-T_{\mathrm{PVbot}}\right)+\frac{A_{\mathrm{PV}}}{2} h c_{\mathrm{PV}, \text { gap }}\left(T_{\text {gap }, \text { bot }}\right.\right.$
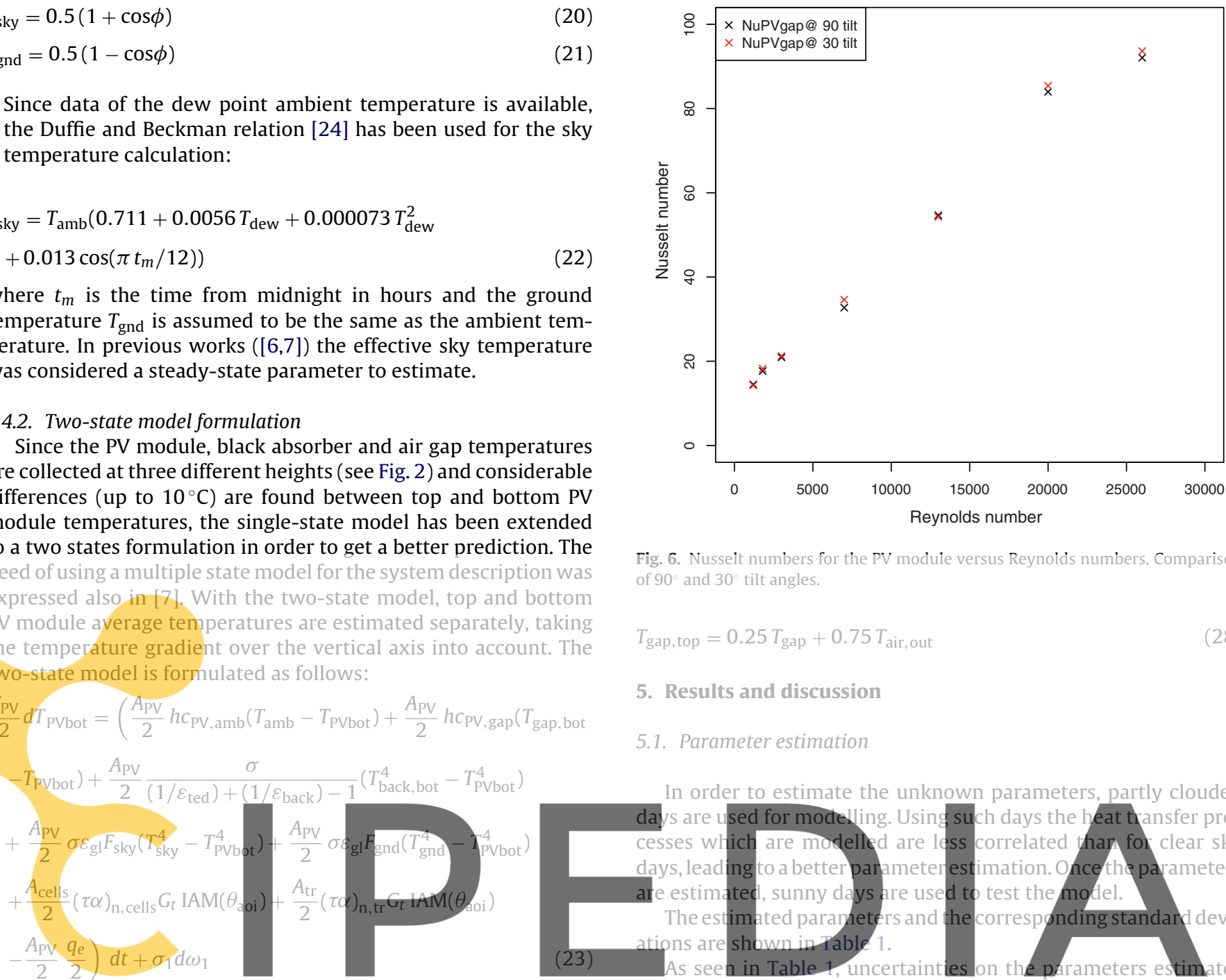

Fig. 6. Nusselt numbers for the PV module versus Reynolds numbers. Comparison of $90^{\circ}$ and $30^{\circ}$ tilt angles

$T_{\text {gap,top }}=0.25 T_{\text {gap }}+0.75 T_{\text {air, out }}$

\section{Results and discussion}

\subsection{Parameter estimation}

In order to estimate the unknown parameters, partly clouded ays are used for modelling. Using such days the heat transfer processes which are modelled are less correlated than for clear sky days, leading to a better parameter estimation. Once the parameters re est The estimated paraneters and the corresponding standard deviAs scions a s seen in Table 1 , uncertainties on the parameters estimates decrease when passing from single to two-state model, confirming the necessity to take the temperature gradient over the vertical axis loakctale version without the watermark

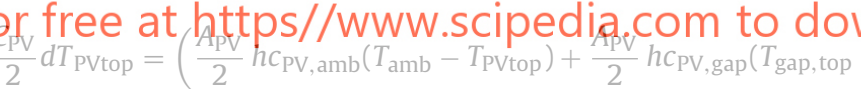

$$
\begin{aligned}
& \left.-T_{\mathrm{PVtop}}\right)+\frac{A_{\mathrm{PV}}}{2} \frac{\sigma}{\left(1 / \varepsilon_{\text {ted }}\right)+\left(1 / \varepsilon_{\text {back }}\right)-1}\left(T_{\text {back,top }}^{4}-T_{\mathrm{PV} \text { top }}^{4}\right) \\
& +\frac{A_{\mathrm{PV}}}{2} \sigma \varepsilon_{\mathrm{gl}} F_{\mathrm{sky}}\left(T_{\text {sky }}^{4}-T_{\mathrm{PVtop}}^{4}\right)+\frac{A_{\mathrm{PV}}}{2} \sigma \varepsilon_{\mathrm{gl}} F_{\mathrm{gnd}}\left(T_{\text {gnd }}^{4}-T_{\mathrm{PVtop}}^{4}\right) \\
& +\frac{A_{\mathrm{cells}}}{2}(\tau \alpha)_{\mathrm{n}, \mathrm{cells}} G_{t} \mathrm{IAM}\left(\theta_{\mathrm{aoi}}\right)+\frac{A_{\mathrm{tr}}}{2}(\tau \alpha)_{\mathrm{n}, \mathrm{tr}} G_{t} \operatorname{IAM}\left(\theta_{\mathrm{aoi}}\right) \\
& \left.-\frac{A_{\mathrm{PV}}}{2} \frac{q_{e}}{2}\right) d t+\sigma_{2} d \omega_{2}
\end{aligned}
$$

$T_{\text {PVbot, } \mathrm{m}}=T_{\text {PVbot }}+e_{1}$

$T_{\mathrm{PVtop}, \mathrm{m}}=T_{\mathrm{PVtop}}+e_{2}$

The model needs 12 inputs $\left(v_{w}, T_{\text {amb }}, T_{\text {gap bot }}, T_{\text {gap,top }}, T_{\text {back,bot }}\right.$, $\left.T_{\text {back,top }}, F_{\text {sky }}, T_{\text {sky }}, F_{\text {gnd }}, \theta_{\text {aoi }}, G_{t}, q_{e}\right), 8$ known parameters $\left(A_{\mathrm{PV}}, A_{\text {cells }}, A_{\mathrm{tr}}, \epsilon_{\mathrm{ted}}, \epsilon_{\mathrm{back}}, \epsilon_{\mathrm{gl}},(\tau \alpha)_{\mathrm{n}, \text { cells }},(\tau \alpha)_{\mathrm{n}, \mathrm{tr}}\right) \quad$ and 4 unknown parameters $\left(C_{\mathrm{PV}}, h c_{\mathrm{PV}, \text { gap }}, \sigma_{1}, \sigma_{2}\right)$ which are estimated.

The same assumptions as for the presented single state model are assumed.

The air gap temperatures are calculated by (see Fig. 5):

$T_{\text {gap, bot }}=0.25 T_{\text {air,in }}+0.75 T_{\text {gap }}$
Starting the evaluation with the estimates of the convective heat transfer coefficients, the average Nusselt numbers are given by:

$\mathrm{Nu}_{\mathrm{PV}, \mathrm{gap}}=\frac{h c_{\mathrm{PV}, \mathrm{gap}} D_{h}}{k_{\mathrm{air}}}$

In Fig. 6, the estimated average Nusselt numbers are shown as a function of the Reynolds number (Re). The tests were performed in vertical position and at $30^{\circ}$ tilt angle and no significant differences are found for the Nusselt numbers. Similar results were found in [14].

Regarding the estimated values of $C_{\mathrm{PV}}$, they differ slightly for distinct Reynolds numbers (see Table 1 ). Taking the uncertainties of the parameters estimate into account, the differences are however not statistically significant for Re $>3000$. For lower Reynolds numbers, the differences could be caused by non-modelled natural convection effects that could be included in more advanced models.

\subsection{Model evaluation}

\subsubsection{Residuals analysis in time and frequency domain}

In Fig. 7 plots of the residuals for the two-state model are shown. The residuals are plotted versus time, solar radiation, state variables and wind speed; these plots may reveal potential outliers or systematic dependencies of the residuals on inputs or states. It 
Table 1

ML estimated parameters and the corresponding standard deviations in vertical position.

\begin{tabular}{|c|c|c|c|c|}
\hline $\begin{array}{l}\text { Model } \\
\text { Ventilation regime }\end{array}$ & $\begin{array}{l}\text { Single-state model } \\
C_{\mathrm{PV}}\end{array}$ & $h c_{\mathrm{PV}, \text { gap }}$ & $\begin{array}{l}\text { Two-state model } \\
C_{\mathrm{PV}}\end{array}$ & $h c_{\mathrm{PV}, \mathrm{gap}}$ \\
\hline $\operatorname{Re}=1200$ & $20823( \pm 1267)$ & $1.56( \pm 0.68)$ & $16504( \pm 281)$ & $1.52( \pm 0.19)$ \\
\hline $\operatorname{Re}=1800$ & - & - & $16020( \pm 151)$ & $2.23( \pm 0.08)$ \\
\hline $\operatorname{Re}=3000$ & $18997( \pm 519)$ & $2.48( \pm 0.08)$ & $17958( \pm 755)$ & $2.52( \pm 0.05)$ \\
\hline $\operatorname{Re}=7000$ & $24262( \pm 1698)$ & $3.94( \pm 0.13)$ & $20788( \pm 650)$ & $4.77( \pm 0.12)$ \\
\hline $\operatorname{Re}=13000$ & $17735( \pm 1615)$ & $6.22( \pm 0.25)$ & $19088( \pm 719)$ & $6.85( \pm 0.16)$ \\
\hline $\operatorname{Re}=20000$ & $23056( \pm 2043)$ & $8.78( \pm 0.30)$ & $22180( \pm 780)$ & $10.78( \pm 0.03)$ \\
\hline $\operatorname{Re}=26000$ & $25924( \pm 1153)$ & $10.38( \pm 0.21)$ & $20278( \pm 484)$ & $11.62( \pm 0.15)$ \\
\hline
\end{tabular}
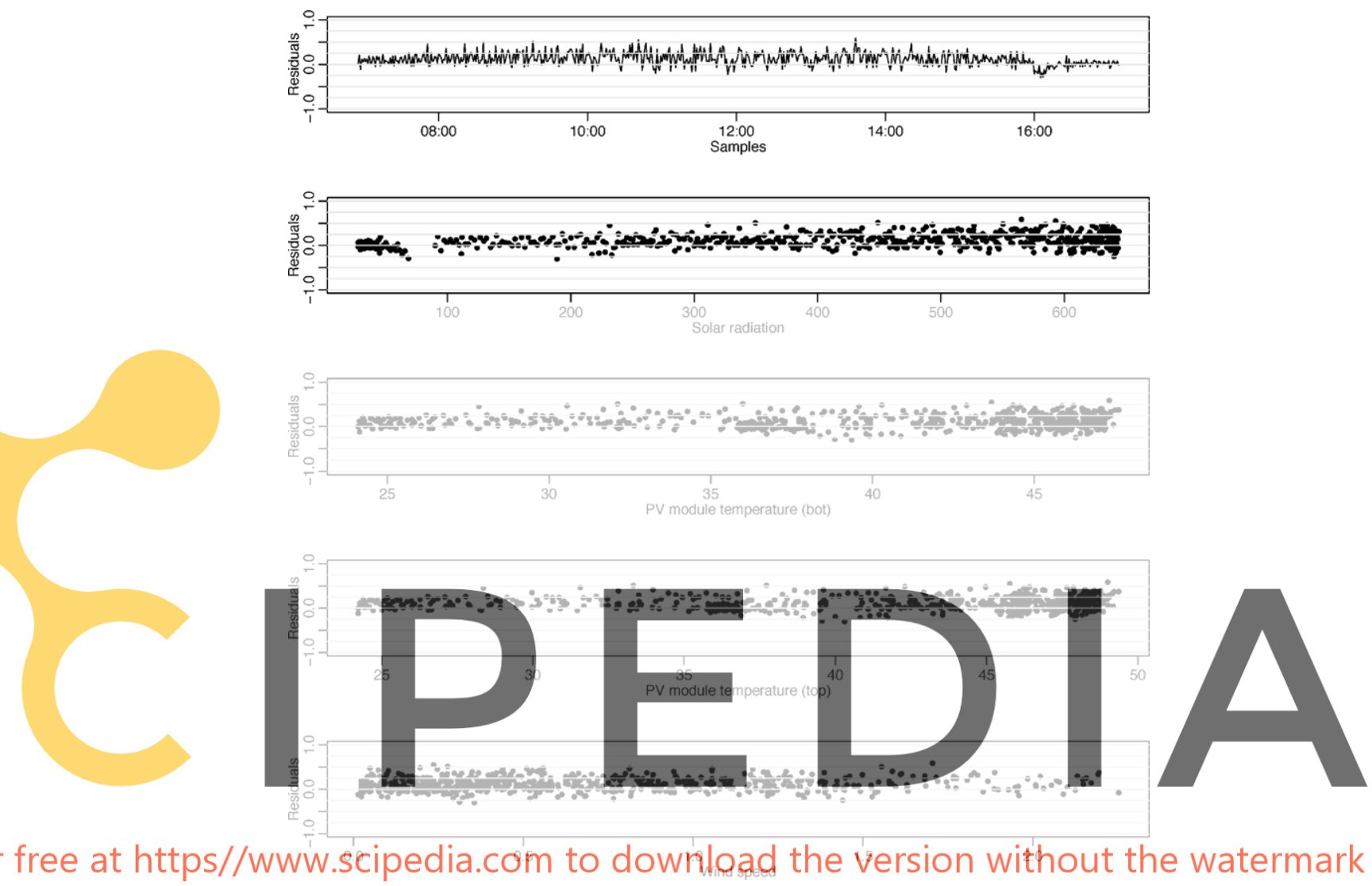

Fig. 7. Plots of the residuals of the two-state model for a one-day period $(\operatorname{Re}=20000)$.

is possible to observe that the variance of the residuals increase slightly with solar radiation and PV module temperature, revealing that a possible model improvement could be the introduction of a dependency of these variables in the noise term of the model. A similar behavior is found also for the single-state model.

To verify that the model describes the dynamics of the system properly, the assumption of white noise residuals (one-step prediction) is checked. The white noise properties are analysed with the auto-correlation functions (ACF) and the cumulated periodograms (CP) plotted in Fig. 8. Confidence bands of approximately 95\% under the hypothesis that the residuals are white noise are also shown. The ACF and the $\mathrm{CP}$ of the residuals clearly show that the two-state model describes the dynamics of the system better than the singlestate model, and in fact it is concluded that a two state is needed to describe all the systematic variations in the data. For the two-state model the white noise assumption of the residuals is not rejected.

\subsubsection{Simulation and prediction}

As a further evaluation, the performance of the two-state model for both simulation and prediction is analysed. Fig. 9 shows simulated and measured $T_{\text {PVtop }}$ for 1 min time step. A similar pattern is found for longer sample periods (i.e. 5 or $10 \mathrm{~min}$ ). The simulated temperatures reasonably follow the observed temperature profile and the standard deviation of the temperature difference is $\left(0.72^{\circ} \mathrm{C}\right)$ for 1 -min data and $\left(0.75^{\circ} \mathrm{C}\right)$ for $10 \mathrm{~min}$ data. The highest temperature differences are registered when there are fast changes in temperature. This is probably due to the fact that some fast dynamical effects are not adequately considered in the model and it reveals that in the simulation, long-time variations are better described than the short-time variations.

The one-step predictions of $T_{\mathrm{PVtop}}$ are compared with the observations in Fig. 10. A similar pattern is found for longer sample periods (i.e. 5 or $10 \mathrm{~min}$ ). It is possible to observe that the model predicts accurately the PV module temperature and the standard deviation of the prediction error is $\left(0.18^{\circ} \mathrm{C}\right)$ for 1 -min data and $\left(0.6^{\circ} \mathrm{C}\right)$ for $10 \mathrm{~min}$ data.

\subsection{Comparison with literature relations for Nusselt numbers in forced convection}

In order to compare the estimated Nusselt numbers with literature relations it is important to define weather the flow is 

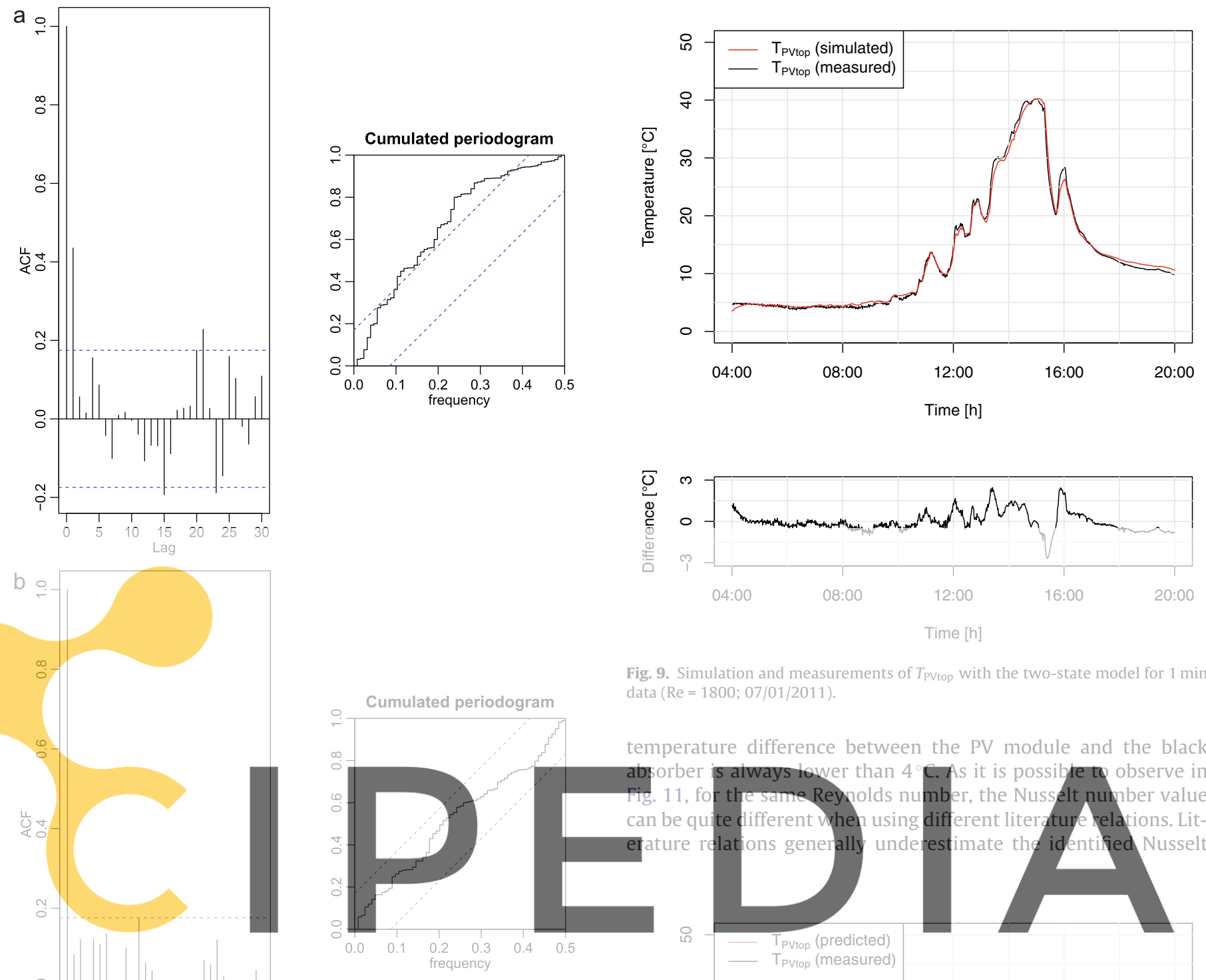

Fig. 9. Simulation and measurements of $T_{\mathrm{PVtop}}$ with the two-state model for $1 \mathrm{~min}$ data $(\operatorname{Re}=1800 ; 07 / 01 / 2011)$

temperature difference between the PV module and the black absorber is aiways tower than 4 C.As it is possible to observe in .11, for the same Reynolds number, the Nusselt number value can be quite different when using different literature relations. Literature relations generally underestimate the identified Nusselt

\section{오}

으 $-T_{\text {PVtop }}$ (predicted)

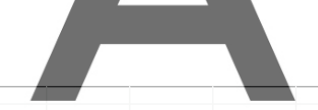

ster for qैree at' https//Www.scipedia.com to downloadothe version without the watermark

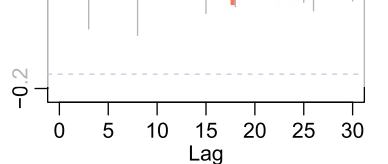

Fig. 8. The auto-correlation function and the cumulated periodogram of the residuals for the selected grey-box model. (a) Single-state model; (b) two-state model.

fully-developed or not. Therefore, the thermal entrance length $\left(x_{\mathrm{th}}\right)$ has been calculated with Bejan [25] relations. In both laminar and turbulent regimes, the fully developed heat transfer condition is not reached, since $x_{\mathrm{th}} \approx 1.7 \mathrm{~m}$ while the air gap length is $1.6 \mathrm{~m}$.

In Fig. 11 the estimated Nusselt numbers are compared with values calculated from some of the most typical literature relations [10-14,26] for developing flow in channels with laminar, transient or turbulent regimes. There are only a few relations for developing flow in transient and turbulent regimes [11-13] because several authors $[25,27]$ assume that in turbulent flow the entrance effects are not significant and that the flow is fully-developed for the entire channel length. Furthermore, literature relations are normally based on isothermal or isoflux boundary conditions but normally in a BIPV system neither isothermal nor isoflux boundary conditions are fulfilled. Relations for both symmetrical and asymmetrical heating are considered in Fig. 11 since the average
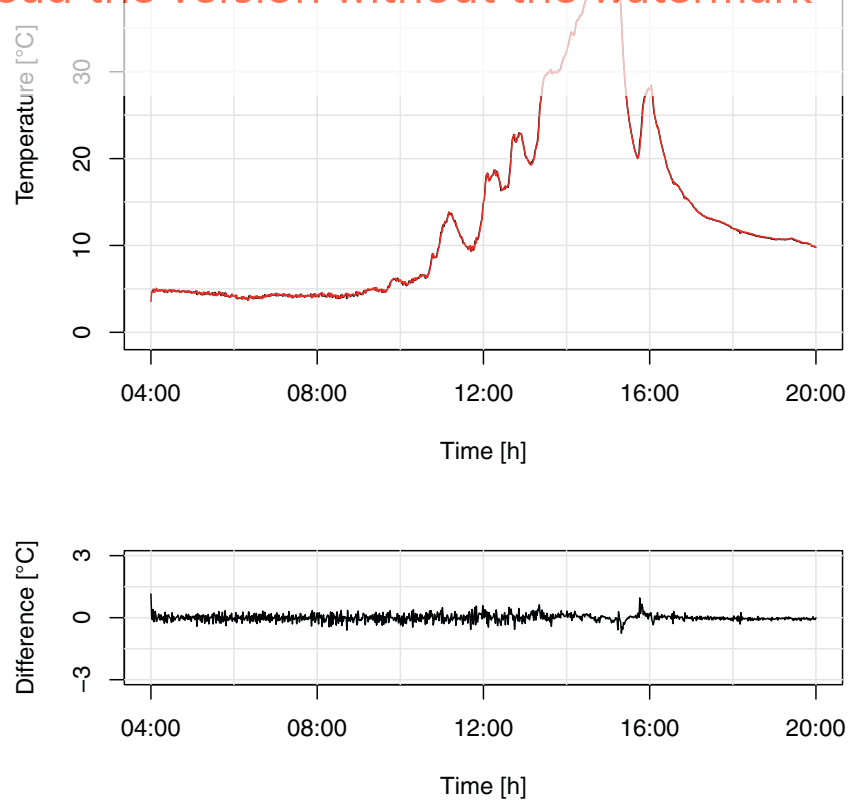

Fig. 10. One-step prediction and measurements of $T_{\mathrm{PV} \text { top }}$ with the two-state model for 1 min data $(\operatorname{Re}=1800 ; 07 / 01 / 2011)$. 


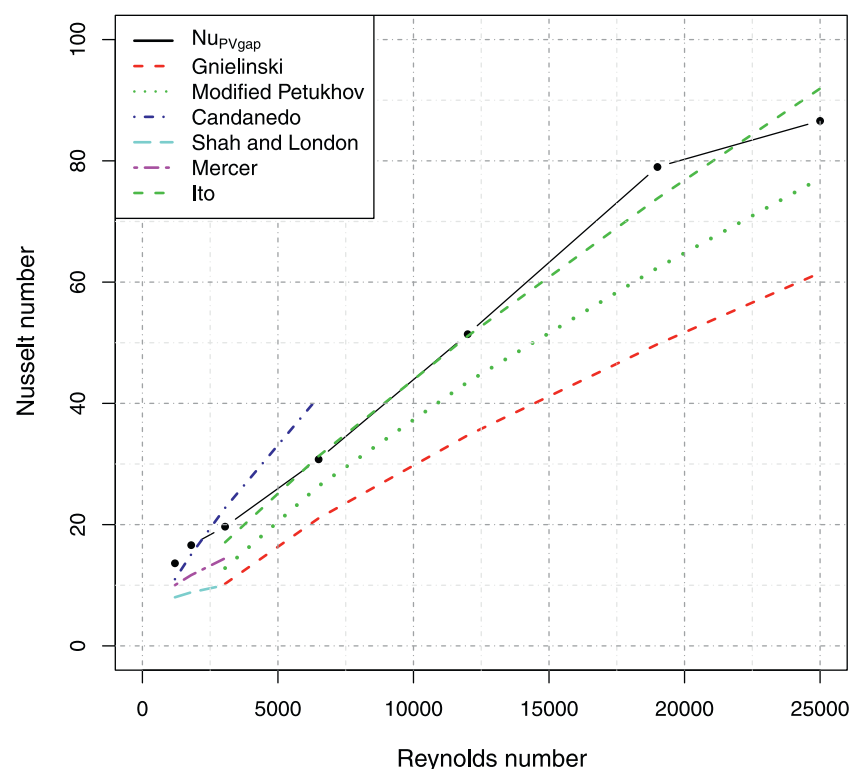

Fig. 11. Comparison of the estimated average Nusselt numbers with values from literature relations.

values. The result is in accordance with several studies on BIPV $[3,14]$. In this particular case, Ito relation [13] provides the best prediction of the estimated Nusselt numbers for transient and turbulent conditions $\left(R^{2}=0.985\right)$. For $R e<3000$, Candanedo's relation predicts quite correctly the estimated values $\left(R^{2}=0.995\right)$.

\section{Conclusions and further developments}

\section{Continuous-discrete} for modelling the heat dy on measured data from a strength of grey-box models and data driven information and providing informationato
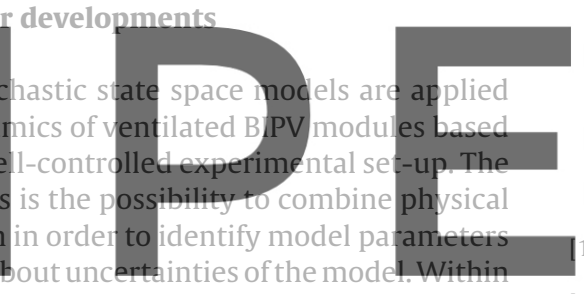

this study, both one-state and two-state grey-box models are presented, and it is shown that the two-state model provides the best

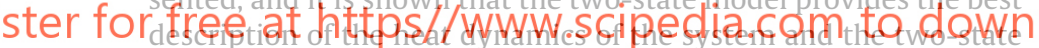
model is not contradicted by white noise tests. This reflects that the second model can be assumed to describe the information in the sampled measurements of the dynamics of the system.

The estimated parameters are evaluated both from a physical and a statistical point of view. Regarding the convective heat transfer coefficients between the PV module and the air gap, it is shown that the most typical literature relations predict quite different values for the same Reynolds number leading to possible calculation errors. In this particular case, the estimated values with the two-state model are predicted properly with the Ito's relation for transient and turbulent regimes, while in laminar conditions Candanedo's relation approximates quite accurately the estimated Nusselt numbers.

Regarding the estimated values of $C_{\mathrm{PV}}$, they slightly differ for different Reynolds number, especially for lower Reynolds numbers; the differences are not statistically significant for $\operatorname{Re}>3000$ and for lower values the difference could be caused by non-modelled natural convection effects that should be included in more advanced models.

The statistical evaluation of the two-state model shows that the model describes the dynamics of the system very well, and that a possible model improvement could be the introduction of a dependency of the solar radiation and the PV module temperature in the noise term.
One promising feature of grey-box models is also the possibility to be applied for simulation and prediction purposes. It is shown that the two-state model performs properly both in prediction and simulation context.

In order to check the reliability and to identify the most suitable model, likelihood ratio tests should be applied in future work [8].

Several rear-facing materials are planned to be tested in the TREL and the applicability of the presented models for different optical properties of the system should be verified in future work.

\section{Acknowledgements}

This work has been financially supported by a FPU program (ref. AP2008-01801) and ENE2010-18357 grants from the Spanish Ministry of Science and Innovation.

\section{References}

[1] D. van Dijk, R. Versluis, PV-HYBRID-PAS: results of thermal performance assessment, in: Proceedings of the 2nd World Conference on Photovoltaic Solar Energy Conversion, Vienna, 1998.

[2] J. Bates, U. Blieske, J. Bloem, J. Campbell, F. Ferrazza, R. Hacker, P. Strachan, Y. Tripanagnostopoulos, Building implementation of photovoltaics with active control of temnerature in. Droceedings of the 17th Euronean Photovoltaic Solar Energy Conference, Munich, 2001.

[3] J. Bloem, Evaluation of a PV-integrated building application in a well-controlled outdoor test environment, Building and Environment 43 (2008) 205-216.

[4] J.J. Bloem, C. Lodi, J. Cipriano, D. Chemisana, An outdoor Test Reference Environment for double skin applications of Building Integrated PhotoVoltaic Systems, Energy and Buildings (2012), http://dx.doi.org/10.1016/j.enbuild.2012.03.023.

5] C. Lodi, J. Cipriano, J. Bloem, D. Chemisana, Design and monitoring of an improved test reference environment for the evaluation of BIPV systems, in: Proceedings of the 25th European Photovoltaic Solar Energy Conference, Valencia, 2010, pp. 5135-5140.

[6] M. Jimenez, H. Madsen, J. Bloem, B. Dammann, Estimation of non-linear continuous time models for the heat exchange dynamics of building integrated

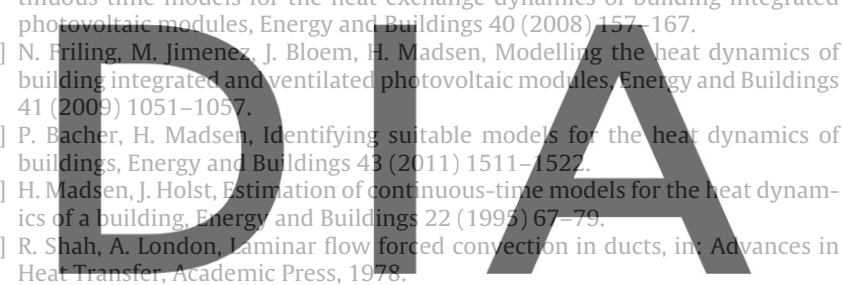

11] F. Incropera, D. De Witt, Fundamentals of Heat and Mass Transfer, John Wiley \& Sons, New York, 1985.

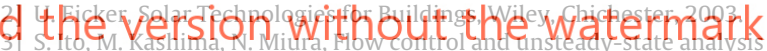
mal performance of solar air collectors, Journal of Solar Energy Engineering 128 (2006) 354-359.

[14] L. Candanedo, A, Athienitis, K, Park, Convective heat transfer coefficients in a building-integrated photovoltaic/thermal system, Journal of Solar Energy Engineering ASME 133 (2011), pp. 021002-1-021002-14.

[15] H. Madsen, J. Holst, E. Lindstrom, Modelling non-linear and non-stationary time series, IMM,DTU (2007).

[16] H. Madsen, Time Series Analysis, Chapman and Hall/CRC, New York, 2008.

[17] H. Madsen, P. Thyregod, Introduction to General and Generalized Linear Models, CRC Press, 2010.

[18] N. Kristensen and H. Madsen, Continuous Time Stochastic Modelling - CTSM 2.3 - Mathematics Guide. 2003.

[19] J. Hirunrabh, W. Kongduang, P. Namprakai, J. Khendari, Study of natural ventilation of houses by a metallic solar wall under tropical climate, Renewable Energy 18 (1999) 109-119.

[20] K. Ong, A mathematical model of a solar chimney, Renewable Energy 28 (2003) 1047-1060.

[21] S. Sharples, Full-scale measurements of convective energy losses from exterior building surfaces, Building and Environment 19 (1984) 31-39.

[22] G. Barker, P. Norton, Building america system performance test practices: Part 1 - photovoltaic systems, NREL/TP-550-30301, National Renewable Energy Laboratory (NREL) (2003).

[23] G. Walton, Thermal Analysis Research Program Reference Manual, No. NBSSIR 83-2655 in National Bureau of Standards (1983).

[24] J.A. Duffie, W.A. Beckman, Solar Engineering of Thermal Processes, 3 ed., Wiley, 2006.

[25] A. Bejan, D. Kraus, Heat Transfer Handbook, John Wiley \& Sons Ltd., 2003.

[26] H. Tan, M. Charters, An experimental investigation of forced convective heat transfer for fully-developed turbulent flow in a rectangular duct with asymmetric heating, Solar Energy 13 (1970) 121-125.

[27] L. Jiji, Heat Convection, Springer ed, 2006. 